\title{
Spectral properties of a symmetric three-dimensional quantum dot with a pair of identical attractive $\delta$-impurities symmetrically situated around the origin
}

\author{
S. Albeverio ${ }^{1,2,3,4}$, S. Fassari ${ }^{2,5,6}$ and F. Rinaldi ${ }^{2,6,7}$ \\ ${ }^{1}$ Institut für Angewandte Mathematik, HCM, IZKS, BiBoS, Universität Bonn, \\ Endenicheralee 60, D-53115 Bonn, Germany \\ ${ }^{2}$ CERFIM, PO Box 1132, CH-6601 Locarno, Switzerland \\ ${ }^{3}$ Centre Interfacultaire Bernoulli, EPFL, CH-1015 Lausanne, Switzerland \\ ${ }^{4}$ Chair Professorship, Department of Mathematics and Statistics, \\ King Fahd University of Petroleum and Minerals, Dhahran, KSA \\ ${ }^{5}$ ISR, Aeulistr.10, CH-9470 Buchs, Switzerland \\ ${ }^{6}$ Università degli Studi Guglielmo Marconi, Via Plinio 44, I-00193 Rome, Italy \\ ${ }^{7}$ BIS Group of Institutions, Gagra-Moga, Punjab under Punjab Technical University, \\ Punjab, India \\ silvestro.fassari@isr.ch
}

\author{
PACS 02.30.Gp, 02.30.Hq, 02.30.Hq, 02.30.Lt, 02.30.Sa, 02.30.Tb, 03.65.Db, \\ 03.65.Ge, 68.65.Hb
}

DOI 10.17586/2220-8054-2016-7-2-268-289

In this presentation, we wish to provide an overview of the spectral features for the self-adjoint Hamiltonian of the three-dimensional isotropic harmonic oscillator perturbed by either a single attractive $\delta$-interaction centered at the origin or by a pair of identical attractive $\delta$-interactions symmetrically situated with respect to the origin. Given that such Hamiltonians represent the mathematical model for quantum dots with sharply localized impurities, we cannot help having the renowned article by Brüning, Geyler and Lobanov [1] as our key reference. We shall also compare the spectral features of the aforementioned three-dimensional models with those of the self-adjoint Hamiltonian of the harmonic oscillator perturbed by an attractive $\delta^{\prime}$-interaction in one dimension, fully investigated in [2,3], given the existence in both models of the remarkable spectral phenomenon called "level crossing". The rigorous definition of the self-adjoint Hamiltonian for the singular double well model will be provided through the explicit formula for its resolvent (Green's function). Furthermore, by studying in detail the equation determining the ground state energy for the double well model, it will be shown that the concept of "positional disorder", introduced in [1] in the case of a quantum dot with a single $\delta$-impurity, can also be extended to the model with the twin impurities in the sense that the greater the distance between the two impurities is, the less localized the ground state will be. Another noteworthy spectral phenomenon will also be determined; for each value of the distance between the two centers below a certain threshold value, there exists a range of values of the strength of the twin point interactions for which the first excited symmetric bound state is more tightly bound than the lowest antisymmetric bound state. Furthermore, it will be shown that, as the distance between the two impurities shrinks to zero, the 3D-Hamiltonian with the singular double well (requiring renormalization to be defined) does not converge to the one with a single $\delta$-interaction centered at the origin having twice the strength, in contrast to its one-dimensional analog for which no renormalization is required. It is worth stressing that this phenomenon has also been recently observed in the case of another model requiring the renormalization of the coupling constant, namely the one-dimensional Salpeter Hamiltonian perturbed by two twin attractive $\delta$-interactions symmetrically situated at the same distance from the origin. 
Keywords: level crossing, degeneracy, point interactions, renormalisation, Schrödinger operators, quantum dots, perturbed quantum oscillators.

Received: 7 November 2015

\section{Introduction}

In this work, we wish to further extend our previous research on various types of point perturbations of Schrödinger Hamiltonians with or without harmonic confinement (see, e.g., [2-11]) to the physical models utilized to describe three-dimensional quantum dots.

This presentation will try to be an expansion of the detailed spectral analysis carried out in [1], in the sense that, after briefly reviewing in Section 2 the findings of [1,9] for the case of a three-dimensional quantum dot with a single impurity consisting of a point interaction centered at the origin, we are going to investigate in Section 3 the model with two twin attractive point impurities symmetrically situated with respect to the bottom of the harmonic confining potential. In Section 2, by analyzing the behavior of the new energy levels created by a point perturbation as functions of the parameter labeling the self-adjoint extensions, physically characterized by being proportional to the inverse scattering length (see [1,4]), we are going to stress the analogy of the presence of the so-called "level crossings" between the newly created eigenenergies of bound states with a given symmetry and those of the unperturbed harmonic oscillator unaffected by the singular perturbation pertaining to bound states having the opposite symmetry. We will also underline the difference between this three-dimensional model and the one-dimensional one, studied in $[2,3]$, in which the harmonic oscillator is perturbed by a so-called $\delta^{\prime}$-interaction, whose quadratic form is given by the expression $\left|\delta^{\prime}\right\rangle\left\langle\delta^{\prime}\right|$.

The first step in the investigation outlined in Section 3 will necessarily be the rigorous definition of the self-adjoint Hamiltonian representing the energy operator for our model. This will be achieved by first introducing the usual ultraviolet energy cut-off and then making the coupling constant dependent on the cut-off itself in such a way that the typical cancellation of divergences will take place in the norm-resolvent limit once the cut-off is removed.

Once the explicit expression of the resolvent (Green's function) is obtained, its poles will provide us with the eigenvalues (energy levels) for the three-dimensional harmonic oscillator perturbed by singular interactions (3D quantum dot with point impurities). The noticeable difference between the bound state equation pertaining to the model with a single impurity centered at the origin (described in Section 2) and the one for the singular double well is the inevitable absence of the simple expression containing the ratio of Gamma functions enabling us to study all the eigenvalues on an equal footing. Although it is still possible, at least conceptually, to carry out the analysis of the entire discrete spectrum, the operational task is made quite challenging by the fact that the equation for an eigenvalue pertaining to a bound state with a given symmetry may not be used for the eigenvalue pertaining to the next upper bound state with the same symmetry without the modifications needed to cancel the inevitable appearance of divergences, a procedure outlined in $[2,8-11]$ in the case of the other aforementioned models.

As a consequence, we have necessarily restricted our task to a rather detailed description of the behavior of the lowest lying eigenvalues (energy levels) of the spectrum with respect to the variations of the two key parameters of the model, one labelling the self-adjoint extensions of the operator (or its reciprocal) and the other one given by the distance between either impurity and the origin.

Given that the findings of our analysis in Section 3 are unavoidably linked to some possible avenues of future research, we refrain from anticipating them in an abbreviated fashion at this stage, as they will be summarized in detail in Section 4, the final section in which 
the most important conclusions will be drawn and some perspectives for future work will be sketched.

\section{The three-dimensional isotropic harmonic oscillator perturbed by a single attractive point perturbation centered at the origin}

We wish to start our analysis by revisiting the model used in [1] to describe a 3Dquantum dot, namely the three-dimensional isotropic harmonic oscillator perturbed by an attractive point perturbation. The spectroscopy of such a Schrödinger Hamiltonian was also fully investigated in [9] under the restriction of having the impurity, that is to say the point perturbation, situated at the origin. Hence, the Hamiltonian was given by the self-adjoint operator making sense of the heuristic expression (see [9]):

$$
H_{\beta}=H_{0}-\beta \delta(\vec{x}), \quad H_{0}=\frac{1}{2} \sum_{i=1}^{3}\left(-\frac{d^{2}}{d x_{i}^{2}}+x_{i}^{2}\right)=\sum_{i=1}^{3} a_{i}^{+} a_{i}+\frac{3}{2}
$$

( $a_{i}$ and its adjoint being the usual annihilation and creation operators associated with $H_{0}$ ).

The eigenfunctions of $H_{0}$, the Hamiltonian of the three-dimensional harmonic oscillator, can clearly be expressed in terms of those of their one-dimensional counterparts, namely:

$$
\Psi_{\vec{n}}(\vec{x})=\psi_{n_{1}}(x) \psi_{n_{2}}(y) \psi_{n_{3}}(z) .
$$

Although, in this case, the radial symmetry of the model could obviously be exploited, as was done in [1], we adhere to the Cartesian framework used in [9] in view of our prospective investigation of the model in which $H_{0}$ is perturbed by two attractive deltas. Before moving forward, it is crucial to notice that the function:

$$
\left(H_{0}-E\right)^{-1}(0, \vec{x})=\sum_{|\vec{n}|=0}^{\infty} \frac{\Psi_{2 \vec{n}}(0)}{\left(|2 \vec{n}|+\frac{3}{2}-E\right)} \Psi_{2 \vec{n}}(\vec{x})
$$

is square integrable. In fact, by considering without loss of generality only negative values of $E$, by means of Parseval's identity, we obtain the following estimate:

$$
\begin{aligned}
\sum_{|\vec{n}|=0}^{\infty} \frac{\Psi_{2 \vec{n}}^{2}(0)}{\left(|2 \vec{n}|+\frac{3}{2}-E\right)^{2}} & \leq \sum_{|\vec{n}|=0}^{\infty} \frac{\Psi_{2 \vec{n}}^{2}(0)}{\left(2 n_{1}+\frac{1}{2}\right)^{2 / 3}\left(2 n_{2}+\frac{1}{2}\right)^{2 / 3}\left(2 n_{3}+\frac{1}{2}\right)^{2 / 3}}= \\
& {\left[\sum_{n=0}^{\infty} \frac{\psi_{2 n}^{2}(0)}{(2 n+1 / 2)^{2 / 3}}\right]^{3}<\infty, }
\end{aligned}
$$

taking into account the well-known pointwise decay of the harmonic oscillator eigenfunctions (see, e.g., $[12,13]$ ), precisely that $\lim _{n \rightarrow \infty} n^{1 / 4} \psi_{n}(x)<\infty$, for any fixed $x$.

As was shown in [9], using the coupling constant renormalization procedure, $H_{\beta}$ is rigorously defined (for any $\beta$ different from zero) by means of its resolvent, namely:

$$
\left(H_{\beta}-E\right)^{-1}=\left(H_{0}-E\right)^{-1}+\frac{\left|\left(H_{0}-E\right)^{-1}(\cdot, 0)\right\rangle\left\langle\left(H_{0}-E\right)^{-1}(0, \cdot)\right|}{\beta^{-1}-E\left[\sum_{|\vec{n}|=0}^{\infty} \frac{\Psi_{2 \vec{n}}^{2}(0)}{\left(|2 \vec{n}|+\frac{3}{2}\right)\left(|2 \vec{n}|+\frac{3}{2}-E\right)}\right]},
$$

for any negative $E$ below $E_{0}(\beta)$, the ground state energy (lowest eigenvalue) of $H_{\beta}$, that is to say the first root of the equation: 


$$
\beta^{-1}=E\left[\sum_{|\vec{n}|=0}^{\infty} \frac{\Psi_{2 \vec{n}}^{2}(0)}{\left(|2 \vec{n}|+\frac{3}{2}\right)\left(|2 \vec{n}|+\frac{3}{2}-E\right)}\right]
$$

as was done in [9] (and in similar contexts in [2, 8, 10,11]), the series on the right hand side of (2.5) can be recast as an integral. This can be achieved by taking advantage of two crucial properties that will be exploited throughout this work: the well-known integral relationship between the resolvent and the semigroup of any semibounded operator for any $E$ below the lowest point in the spectrum (see, e.g., [14] page 204), as well as the fact that the integral kernel of the semigroup of the three-dimensional harmonic oscillator is perfectly separable (see [1] page 1278), such that (2.5) reads for any $E<3 / 2$ :

$$
\beta^{-1}=\frac{1}{\sqrt{\pi^{3}}} \int_{0}^{\infty} \frac{e^{\frac{3}{2} t}\left(e^{E t}-1\right)}{\left(e^{2 t}-1\right)^{\frac{3}{2}}} d t
$$

or equivalently,

$$
\beta^{-1}=\frac{1}{\sqrt{\pi^{3}}} \int_{0}^{\infty} \frac{e^{-\frac{3}{2} t}\left(e^{E t}-1\right)}{\left(1-e^{-2 t}\right)^{\frac{3}{2}}} d t
$$

By means of a simple change of variable inside the latter integral, $(2.5 \mathrm{~b})$ can be rewritten as an integral over a bounded interval (involving Mehler's kernel):

$$
\beta^{-1}=\frac{1}{\sqrt{\pi^{3}}} \int_{0}^{1} \frac{\xi^{\frac{1}{2}}\left(\xi^{-E}-1\right)}{\left(1-\xi^{2}\right)^{\frac{3}{2}}} d \xi
$$

By setting $\alpha=1 / \beta$ and

$$
\alpha_{0}=\beta_{0}^{-1}=\frac{1}{\sqrt{\pi^{3}}} \int_{0}^{1} \frac{1-\xi^{\frac{1}{2}}}{\left(1-\xi^{2}\right)^{\frac{3}{2}}} d \xi=\frac{\Gamma(3 / 4)}{\pi \cdot \Gamma(1 / 4)} \cong 0.107585,
$$

(2.5c) can be further transformed into:

$$
\alpha-\alpha_{0}=\frac{1}{\sqrt{\pi^{3}}} \int_{0}^{1} \frac{\xi^{\frac{1}{2}-E}-1}{\left(1-\xi^{2}\right)^{3} / 2} d \xi .
$$

In perfect analogy with what was done in the aforementioned articles, the integral on the right hand side can be converted into a ratio of Gamma functions as follows:

$$
\alpha_{0}-\alpha=\frac{\Gamma\left(\frac{3}{4}-\frac{E}{2}\right)}{\pi \Gamma\left(\frac{1}{4}-\frac{E}{2}\right)} .
$$

It is crucial to realize that $(2.5 \mathrm{e})$ holds also to the right of $E=3 / 2$, enabling us to determine all the eigenvalues and not only the ground state energy. Of course, our analysis in this work is entirely consistent with what was observed in [9] (see the final remark in [2] as well). The graph of the ground state energy as a function of the extension parameter $\alpha=1 / \beta$ is shown below (Fig. 1). As is evident, $E_{0}(\alpha) \rightarrow 3 / 2$ as $\alpha \rightarrow+\infty$. 


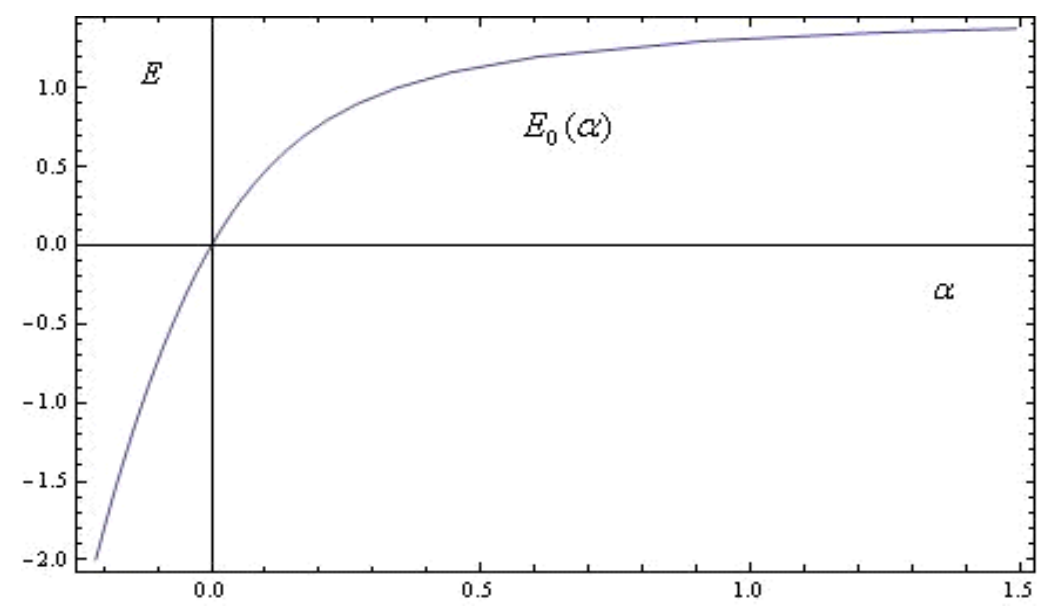

FIG. 1. The ground state energy of the 3D-isotropic harmonic oscillator perturbed by an attractive point interaction situated at the origin as a function of the extension parameter $\alpha=1 / \beta$

At this stage, it might be worth recalling that the corresponding bound state equation for the one-dimensional Hamiltonian:

$$
h(\alpha)=h_{1 / \alpha}=\frac{1}{2}\left(-\frac{d^{2}}{d x^{2}}+x^{2}\right)-\frac{1}{\alpha}\left|\delta^{\prime}(x)\right\rangle\left\langle\delta^{\prime}(x)\right|,
$$

defined rigorously by means of its resolvent in two different ways in [2,3], is instead:

$$
\alpha_{0}^{1 D}-\alpha=2 \frac{\Gamma\left(\frac{3}{4}-\frac{E}{2}\right)}{\Gamma\left(\frac{1}{4}-\frac{E}{2}\right)}, \quad \alpha_{0}^{1 D}=2 \frac{\Gamma(3 / 4)}{\Gamma(1 / 4)} \cong 0.675978 .
$$

Conversely, the corresponding bound state equation for the one-dimensional Hamiltonian is:

$$
h(\lambda)=\frac{1}{2}\left(-\frac{d^{2}}{d x^{2}}+x^{2}\right)-\lambda \delta(x)=\frac{1}{2}\left(-\frac{d^{2}}{d x^{2}}+x^{2}\right)-\lambda|\delta(x)\rangle\langle\delta(x)|,
$$

taking account of the obvious identity in the sense of quadratic forms between the $\delta$-interaction, namely the quadratic form $|\delta\rangle\langle\delta|$, and the so-called $\delta$-potential (whose quadratic form is instead $(\cdot, \delta \cdot))$, reads:

(see $[2,8,15])$.

$$
\lambda=2 \frac{\Gamma\left(\frac{3}{4}-\frac{E}{2}\right)}{\Gamma\left(\frac{1}{4}-\frac{E}{2}\right)}
$$

The fact that (2.7), unlike (2.5e) and (2.6), involves only the coupling constant is a clear manifestation of the fact that $h(\lambda)$ can be easily defined by means of the KLMN theorem (see [16]) without the renormalization required for the other two models.

The higher energy levels are even more interesting, as they exhibit the phenomenon called "level crossing". As was pointed out in [2] (see also a similar remark in [9]): "as a consequence of the presence of the point perturbation acting only on states with zero angular momentum, the $\frac{(2 l+1)(2 l+2)}{2}$-degeneracy of the eigenvalue $E_{2 l}=2 l+\frac{3}{2}$ gets lowered by one due to the emergence of the simple eigenvalue generated by the perturbation. Such a simple eigenvalue, regarded as a function of the extension parameter $\alpha$, does cross the next lower unperturbed eigenvalue $E_{2 l-1}=(2 l-1)+\frac{3}{2}$," 
As can be seen in Fig. 2, it is quite noteworthy that all these level crossings take place exactly at $\alpha=\alpha_{0}$ (approximately equal to 0.107585 ), adopting the renormalization used in [9]. As was stressed in [2]:

"by using instead the alternative renormalization (3.6), the location of all the level crossings would be exactly $\tilde{\alpha}^{(3)}=0$, leading to the graph shown in Fig. 4(a) of the aforementioned paper by Brüning, Geyler and Lobanov".

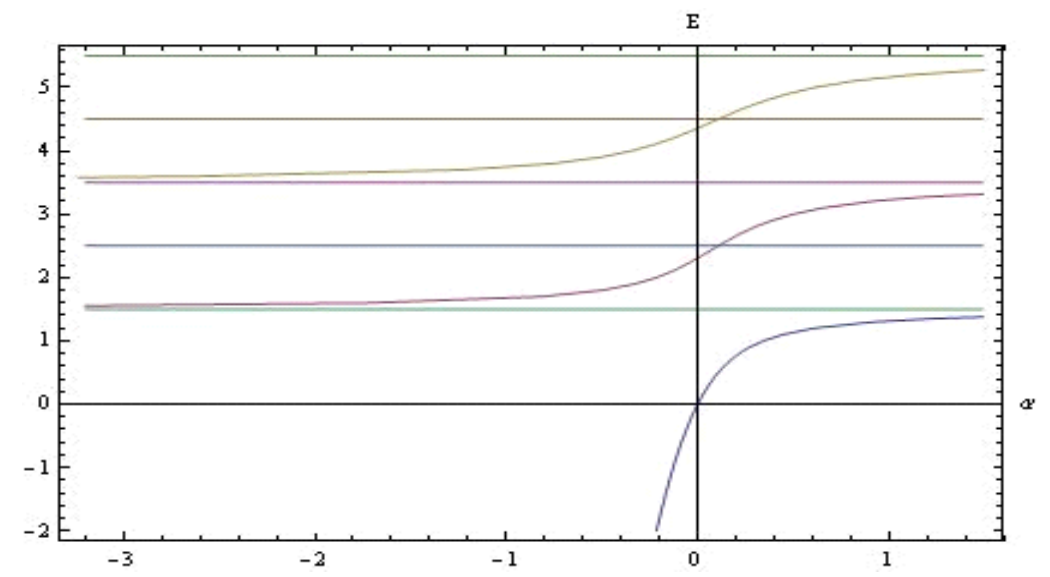

FIG. 2. The ground state energy and the next two symmetric eigenenergies of the $3 \mathrm{D}$-isotropic harmonic oscillator with an attractive $\delta$-perturbation situated at the origin as functions of the extension parameter $\alpha=1 / \beta$

The very same phenomenon observed in [2] for the one-dimensional Hamiltonian $h(\alpha)$, for which all the level crossings take place exactly at $\alpha=\alpha_{0}^{(1 D)}$ (approximately equal to 0.675978) using the renormalization (2.4a) of that paper, is to be regarded as being even more remarkable. Citing again our considerations in [2], it can be stated that:

"from the point of view of the structure of the spectral curves representing the eigenvalues (energy levels) as functions of the extension parameter and neglecting the degeneracy of the three-dimensional eigenvalues, the harmonic oscillator perturbed by the point interaction considered here $\left(\left|\delta^{\prime}(x)\right\rangle\left\langle\delta^{\prime}(x)\right|\right)$ seems to be a more legitimate one-dimensional counterpart than the delta distribution as the latter bears no resemblance of the $3 D$-level crossing involving eigenstates of different symmetry. Having stated that analogy, an important difference must also be pointed out: whilst in the case of the perturbed isotropic oscillator the eigenenergy of the simple eigenvalue created by the point interaction (emerging out of a degenerate level with an even value of the total angular momentum) can cross the next lower unperturbed level (having an odd value of the total angular momentum) beyond a certain threshold of the key parameter of that model, something of an opposite nature occurs in the one-dimensional model being studied here: each perturbed odd eigenvalue can fall below the next lower unperturbed even eigenvalue beyond a certain threshold. As a consequence, the symmetry of the ground state wave function can change in the case of a sufficiently strong $\delta^{\prime}$-interaction (the ground state wave function being given by an odd function discontinuous at the origin)".

The spectral features of the Hamiltonian for our perturbed isotropic oscillator described in the previous remarks can be visualized in the above graph (Fig. 2) displaying the new ground state energy $E_{0}(\alpha)$ and those of the two symmetric bound states $E_{2}(\alpha)$ and $E_{4}(\alpha)$ which emerge from the $7 / 2$ and $11 / 2$ levels as a result of the perturbation and are confined inside the intervals $(3 / 2,7 / 2)$ and $(7 / 2,11 / 2)$, as functions of the extension parameter, as well as their level crossings with the unperturbed antisymmetric states $E=5 / 2$ and $E=9 / 2$. Both of these 
occur, as anticipated above, at $\alpha=\alpha_{0}$ (approximately equal to 0.107585 ). It is worth reminding the reader that the degeneracy of $E=7 / 2$ has been lowered to five and that of $E=11 / 2$ to fourteen.

\section{The three-dimensional isotropic harmonic oscillator perturbed by two twin attractive point perturbations symmetrically situated with respect to the origin}

We wish to continue our analysis of the three-dimensional model by considering the heuristic Hamiltonian:

$$
H_{\left\{\beta, \vec{x}_{0}\right\}}=H_{0}-\beta\left[\delta\left(\vec{x}-\vec{x}_{0}\right)+\delta\left(\vec{x}+\vec{x}_{0}\right)\right], \quad \vec{x}_{0}=\left(x_{0}, 0,0\right), \quad x_{0}>0 .
$$

As pointed out in the final remarks in [17] (see also the references therein), the model with a singular double well has been increasingly utilized in the recent condensed matter theory literature "since, in combination with a cubic nonlinearity, it leads to a particular type of the nonlinear Schrödinger equation for Bose-Einstein condensates (Gross-Pitaevskii equation)".

By essentially mimicking what was done in $[2,9]$, we can start by performing the standard ultraviolet cut-off and making the coupling $\mu=\mu(\ell, \beta)>0$ dependent upon the related upper limit $0<\ell<+\infty$ for the energy, which leads to the introduction of a perfectly defined self-adjoint Hamiltonian:

$$
H_{\left\{\ell, \beta, \vec{x}_{0}\right\}}=H_{0}-\mu(\ell, \beta) \sum_{|\vec{l}|=0,|\vec{m}|=0}^{\ell}\left|\Psi_{\vec{l}}\right\rangle\left[\Psi_{\vec{l}}\left(\vec{x}_{0}\right) \Psi_{\vec{m}}\left(\vec{x}_{0}\right)+\Psi_{\vec{l}}\left(-\vec{x}_{0}\right) \Psi_{\vec{m}}\left(-\vec{x}_{0}\right)\right]\left\langle\Psi_{\vec{m}}\right|,
$$

which implies that for any sufficiently negative $E$ we can write:

$$
\begin{aligned}
H_{\left\{\ell, \beta, \vec{x}_{0}\right\}}-E= & \left(H_{0}-E\right)^{1 / 2}\left\{I-\mu(\ell, \beta)\left[\left|\left(H_{0}^{\ell}-E\right)^{-1 / 2}\left(\cdot,-\vec{x}_{0}\right)\right\rangle\left\langle\left(H_{0}^{\ell}-E\right)^{-1 / 2}\left(-\vec{x}_{0}, \cdot\right)\right|+\right.\right. \\
& \left.\left.\left|\left(H_{0}^{\ell}-E\right)^{-1 / 2}\left(\cdot, \vec{x}_{0}\right)\right\rangle\left\langle\left(H_{0}^{\ell}-E\right)^{-1 / 2}\left(\vec{x}_{0}, \cdot\right)\right|\right]\right\}\left(H_{0}-E\right)^{1 / 2} .
\end{aligned}
$$

Therefore, in order to explicitly write the resolvent of the left hand side of (3.2), it is crucial to determine the inverse of the operator inside the curly brackets on the right hand side of (3.3). We wish to remind the reader that the expression of the resolvent given by the inverse of (3.3) is known as Tiktopoulos' formula, commonly used in dealing with perturbations of semibounded operators (see, e.g., Theor. VIII.25 in [18]). It is worth noting that in the latter expansion of the resolvent the crucial role is played by an operator of the type:

$$
(A+I)^{-1 / 2}\left(A_{n}-A\right)(A+I)^{-1 / 2}, \quad A \geq 0,
$$

which is isospectral to the more widely investigated Birman-Schwinger operator (see, e.g., [19, $20]$ in the case of perturbations of the negative Laplacian in one dimension). In perfect analogy with what was done in $[7,11,17]$, it is possible to rewrite the operator of rank two inside the square brackets on the right hand side of (3.3) as follows:

$$
\begin{aligned}
& \left|\left(H_{0}^{\ell}-E\right)^{-1 / 2}\left(\cdot,-\vec{x}_{0}\right)\right\rangle\left\langle\left(H_{0}^{\ell}-E\right)^{-1 / 2}\left(-\vec{x}_{0}, \cdot\right)|+|\left(H_{0}^{\ell}-E\right)^{-1 / 2}\left(\cdot, \vec{x}_{0}\right)\right\rangle\left\langle\left(H_{0}^{\ell}-E\right)^{-1 / 2}\left(\vec{x}_{0}, \cdot\right)\right|= \\
& 2\left[\left|\left(H_{0}^{\ell}-E\right)_{s}^{-1 / 2}\left(\cdot, \vec{x}_{0}\right)\right\rangle\left\langle\left(H_{0}^{\ell}-E\right)_{s}^{-1 / 2}\left(\vec{x}_{0}, \cdot\right)|+|\left(H_{0}^{\ell}-E\right)_{a s}^{-1 / 2}\left(\cdot, \vec{x}_{0}\right)\right\rangle\left\langle\left(H_{0}^{\ell}-E\right)_{a s}^{-1 / 2}\left(\vec{x}_{0}, \cdot\right)\right|\right],
\end{aligned}
$$

where

$$
\begin{gathered}
\left(H_{0}^{\ell}-E\right)_{s}^{-1 / 2}\left(\vec{x}_{0}, \vec{x}\right):=\sum_{|\vec{n}|=0}^{\ell} \frac{\Psi_{2 \vec{n}}\left(\vec{x}_{0}\right)}{\left(|2 \vec{n}|+\frac{3}{2}-E\right)^{1 / 2}} \Psi_{2 \vec{n}}(\vec{x}) \\
\left(H_{0}^{\ell}-E\right)_{a s}^{-1 / 2}\left(\vec{x}_{0}, \vec{x}\right):=
\end{gathered}
$$




$$
\sum_{|\vec{n}|=0}^{\ell} \frac{\psi_{2 n_{1}+1}\left(x_{0}\right) \psi_{2 n_{2}}(0) \psi_{2 n_{3}}(0)}{\left(2 n_{1}+2 n_{2}+2 n_{3}+\frac{5}{2}-E\right)^{1 / 2}} \psi_{2 n_{1}+1}(x) \psi_{2 n_{2}}(y) \psi_{2 n_{3}}(z),
$$

which implies their mutual orthogonality.

By essentially mimicking what was done in the aforementioned papers, it is rather straightforward to obtain the algebra for the powers of the rank-two operator on the right hand side of (3.4):

$$
\begin{gathered}
\left\{2 \left[\left|\left(H_{0}^{\ell}-E\right)_{s}^{-1 / 2}\left(\cdot, \vec{x}_{0}\right)\right\rangle\left\langle\left(H_{0}^{\ell}-E\right)_{s}^{-1 / 2}\left(\vec{x}_{0}, \cdot\right)\right|+\right.\right. \\
\left.\left.\left|\left(H_{0}^{\ell}-E\right)_{a s}^{-1 / 2}\left(\cdot, \vec{x}_{0}\right)\right\rangle\left\langle\left(H_{0}^{\ell}-E\right)_{a s}^{-1 / 2}\left(\vec{x}_{0}, \cdot\right)\right|\right]\right\}^{m+1}= \\
2^{m+1}\left\{\left[\left(H_{0}^{\ell}-E\right)_{s}^{-1}\left(\vec{x}_{0}, \vec{x}_{0}\right)\right]^{m}\left|\left(H_{0}^{\ell}-E\right)_{s}^{-1 / 2}\left(\cdot, \vec{x}_{0}\right)\right\rangle\left\langle\left(H_{0}^{\ell}-E\right)_{s}^{-1 / 2}\left(\vec{x}_{0}, \cdot\right)\right|+\right. \\
\left.\left[\left(H_{0}^{\ell}-E\right)_{a s}^{-1}\left(\vec{x}_{0}, \vec{x}_{0}\right)\right]^{m}\left|\left(H_{0}^{\ell}-E\right)_{a s}^{-1 / 2}\left(\cdot, \vec{x}_{0}\right)\right\rangle\left\langle\left(H_{0}^{\ell}-E\right)_{a s}^{-1 / 2}\left(\vec{x}_{0}, \cdot\right)\right|\right\},
\end{gathered}
$$

given that:

$$
\left\|\left(H_{0}^{\ell}-E\right)_{s}^{-1 / 2}\left(\vec{x}_{0}, \cdot\right)\right\|_{2}^{2}=\sum_{|\vec{n}|=0}^{\ell} \frac{\Psi_{2 \vec{n}}^{2}\left(\vec{x}_{0}\right)}{|2 \vec{n}|+\frac{3}{2}-E}=\left(H_{0}^{\ell}-E\right)_{s}^{-1}\left(\vec{x}_{0}, \vec{x}_{0}\right),
$$

and similarly for the square of the norm of $\left(H_{0}^{\ell}-E\right)_{a s}^{-1 / 2}\left(\vec{x}_{0}, \cdot\right)$. Hence, the inverse of the operator inside the curly brackets of (3.3) can be written as a new rank-two operator:

$$
\begin{aligned}
& \frac{1}{\frac{1}{2 \mu(\ell, \beta)}-\left(H_{0}^{\ell}-E\right)_{s}^{-1}\left(\vec{x}_{0}, \vec{x}_{0}\right)}\left|\left(H_{0}^{\ell}-E\right)_{s}^{-1 / 2}\left(\cdot, \vec{x}_{0}\right)\right\rangle\left\langle\left(H_{0}^{\ell}-E\right)_{s}^{-1 / 2}\left(\vec{x}_{0}, \cdot\right)\right|+ \\
& \frac{1}{\frac{1}{2 \mu(\ell, \beta)}-\left(H_{0}^{\ell}-E\right)_{a s}^{-1}\left(\vec{x}_{0}, \vec{x}_{0}\right)}\left|\left(H_{0}^{\ell}-E\right)_{a s}^{-1 / 2}\left(\cdot, \vec{x}_{0}\right)\right\rangle\left\langle\left(H_{0}^{\ell}-E\right)_{a s}^{-1 / 2}\left(\vec{x}_{0}, \cdot\right)\right| .
\end{aligned}
$$

Therefore, the inverse of the operator on the left hand side of (3.3) is:

$$
\begin{aligned}
& \left(H_{\left\{\ell, \beta, \vec{x}_{0}\right\}}-E\right)^{-1}=\left(H_{0}-E\right)^{-1}+ \\
& \frac{1}{\frac{1}{2 \mu(\ell, \beta)}-\left(H_{0}^{\ell}-E\right)_{s}^{-1}\left(\vec{x}_{0}, \vec{x}_{0}\right)}\left|\left(H_{0}^{\ell}-E\right)_{s}^{-1}\left(\cdot, \vec{x}_{0}\right)\right\rangle\left\langle\left(H_{0}^{\ell}-E\right)_{s}^{-1}\left(\vec{x}_{0}, \cdot\right)\right|+ \\
& \frac{1}{\frac{1}{2 \mu(\ell, \beta)}-\left(H_{0}^{\ell}-E\right)_{a s}^{-1}\left(\vec{x}_{0}, \vec{x}_{0}\right)}\left|\left(H_{0}^{\ell}-E\right)_{a s}^{-1}\left(\cdot, \vec{x}_{0}\right)\right\rangle\left\langle\left(H_{0}^{\ell}-E\right)_{a s}^{-1}\left(\vec{x}_{0}, \cdot\right)\right| .
\end{aligned}
$$

At this stage, we need to thoroughly investigate what occurs when the ultraviolet cut-off gets removed, that is to say the behavior of (3.7) as $\ell \rightarrow+\infty$.

As a consequence of the simple estimate:

$$
\begin{aligned}
\sum_{|\vec{n}|=0}^{\ell} \frac{\Psi_{2 \vec{n}}^{2}\left(\vec{x}_{0}\right)}{\left(|2 \vec{n}|+\frac{3}{2}-E\right)^{2}} \leq \sum_{|\vec{n}|=0}^{\infty} \frac{\Psi_{2 \vec{n}}^{2}\left(\vec{x}_{0}\right)}{\left(|2 \vec{n}|+\frac{3}{2}-E\right)^{2}} \leq \\
\quad\left[\sum_{n=0}^{\infty} \frac{\psi_{2 n}^{2}\left(x_{0}\right)}{(2 n+1 / 2)^{2 / 3}}\right]\left[\sum_{n=0}^{\infty} \frac{\psi_{2 n}^{2}(0)}{(2 n+1 / 2)^{2 / 3}}\right]^{2}<\infty
\end{aligned}
$$


it is immediate to realize that $\left(H_{0}^{\ell}-E\right)_{s}^{-1}\left(\vec{x}_{0}, \cdot\right)$ will converge in the Hilbert space norm to the following:

$$
\left(H_{0}-E\right)_{s}^{-1}\left(\vec{x}_{0}, \vec{x}\right):=\sum_{|\vec{n}|=0}^{\infty} \frac{\Psi_{2 \vec{n}}\left(\vec{x}_{0}\right)}{|2 \vec{n}|+\frac{3}{2}-E} \Psi_{2 \vec{n}}(\vec{x}) .
$$

The latter convergence implies in a straightforward manner that the rank-one operator:

$$
\left|\left(H_{0}^{\ell}-E\right)_{s}^{-1}\left(\cdot, \vec{x}_{0}\right)\right\rangle\left\langle\left(H_{0}^{\ell}-E\right)_{s}^{-1}\left(\vec{x}_{0}, \cdot\right)\right|,
$$

will converge in the trace class norm to the following:

$$
\left|\left(H_{0}-E\right)_{s}^{-1}\left(\cdot, \vec{x}_{0}\right)\right\rangle\left\langle\left(H_{0}-E\right)_{s}^{-1}\left(\vec{x}_{0}, \cdot\right)\right| .
$$

Similarly, it follows that:

$$
\left|\left(H_{0}^{\ell}-E\right)_{a s}^{-1}\left(\cdot, \vec{x}_{0}\right)\right\rangle\left\langle\left(H_{0}^{\ell}-E\right)_{a s}^{-1}\left(\vec{x}_{0}, \cdot\right)|\rightarrow|\left(H_{0}-E\right)_{a s}^{-1}\left(\cdot, \vec{x}_{0}\right)\right\rangle\left\langle\left(H_{0}-E\right)_{a s}^{-1}\left(\vec{x}_{0}, \cdot\right)\right|,
$$

with

$$
\left(H_{0}-E\right)_{a s}^{-1}\left(\vec{x}_{0}, \vec{x}\right):=\sum_{|\vec{n}|=0}^{\infty} \frac{\psi_{2 n_{1}+1}\left(x_{0}\right) \psi_{2 n_{2}}(0) \psi_{2 n_{3}}(0)}{\left(2 n_{1}+2 n_{2}+2 n_{3}+\frac{5}{2}-E\right)^{1 / 2}} \psi_{2 n_{1}+1}(x) \psi_{2 n_{2}}(y) \psi_{2 n_{3}}(z)
$$

Therefore, the only problem caused by the removal of the ultraviolet cut-off is the divergence of the series inside each denominator in the second and third term on the right hand side of (3.7). However, we still have the freedom of choosing the $\ell$-dependence of $\mu(\ell, \beta)$ in such a way that the typical "cancellation of infinities" may take place. Let us set:

$$
\frac{1}{\mu(\ell, \beta)}=\frac{1}{\beta}+\sum_{|\vec{n}|=0}^{\ell} \frac{\Psi_{2 \vec{n}}^{2}(0)}{|2 \vec{n}|+\frac{3}{2}},
$$

or equivalently:

$$
\mu(\ell, \beta)=\beta\left[1+\beta \sum_{|\vec{n}|=0}^{\ell} \frac{\Psi_{2 \vec{n}}^{2}(0)}{|2 \vec{n}|+\frac{3}{2}}\right]^{-1} .
$$

In perfect accordance with the use of the term "attractive" in $[2,3,9,17]$, it is clear that $\mu(\ell, \beta)>0$ for the large values of $\ell$ involved in the limit, regardless of the sign of $\beta$. This makes the singular interaction attractive because of the presence of the negative sign in the second term in (3.2). Hence, for any $E<3 / 2$ :

$$
\frac{1}{2 \mu(\ell, \beta)}-\left(H_{0}^{\ell}-E\right)_{s}^{-1}\left(\vec{x}_{0}, \vec{x}_{0}\right)=\frac{1}{2 \beta}+\frac{1}{2} \sum_{|\vec{n}|=0}^{\ell} \frac{\Psi_{2 \vec{n}}^{2}(0)}{|2 \vec{n}|+\frac{3}{2}}-\sum_{|\vec{n}|=0}^{\ell} \frac{\Psi_{2 \vec{n}}^{2}\left(\vec{x}_{0}\right)}{|2 \vec{n}|+\frac{3}{2}-E},
$$

and

$$
\begin{aligned}
& \frac{1}{2 \mu(\ell, \beta)}-\left(H_{0}^{\ell}-E\right)_{a s}^{-1}\left(\vec{x}_{0}, \vec{x}_{0}\right)= \\
& \frac{1}{2 \beta}+\frac{1}{2} \sum_{|\vec{n}|=0}^{\ell} \frac{\Psi_{2 \vec{n}}^{2}(0)}{|2 \vec{n}|+\frac{3}{2}}-\sum_{|\vec{n}|=0}^{\ell} \frac{\psi\left(x_{0}\right)_{2 n_{1}+1}^{2} \psi_{2 n_{2}}^{2}(0) \psi_{2 n_{3}}^{2}(0)}{2 n_{1}+2 n_{2}+2 n_{3}+\frac{5}{2}-E},
\end{aligned}
$$

(as will be seen shortly, considered separately, the latter expression is well defined for any $E<5 / 2)$.

By taking advantage of the integral representation for the resolvent of the three-dimensional harmonic oscillator for any $E<3 / 2$, that is to say the aforementioned Mehler's kernel, as well 
as of the explicit expressions of the symmetric and antisymmetric components of the integral kernel of the semigroup for the one-dimensional harmonic oscillator established in [11], the limit of the right hand side of (3.12), as $\ell \rightarrow+\infty$ can be written as:

$$
\begin{array}{r}
\frac{1}{2 \beta}+\frac{1}{2 \pi^{3 / 2}}\left[\int_{0}^{1} \frac{\xi^{\frac{1}{2}}}{\left(1-\xi^{2}\right)^{3 / 2}} d \xi-\int_{0}^{1} \frac{\xi^{\frac{1}{2}-E}\left[e^{-x_{0}^{2} \frac{1-\xi}{1+\xi}}+e^{-x_{0}^{2} \frac{1+\xi}{1-\xi}}\right]}{\left(1-\xi^{2}\right)^{3 / 2}} d \xi\right]= \\
\frac{1}{2 \beta}+\frac{1}{2 \pi^{3 / 2}} \int_{0}^{1} \frac{\xi^{1 / 2}\left[1-\xi^{-E}\left(e^{-x_{0}^{2} \frac{1-\xi}{1+\xi}}+e^{-x_{0}^{2} \frac{1+\xi}{1-\xi}}\right)\right]}{\left(1-\xi^{2}\right)^{3 / 2}} d \xi<\infty .
\end{array}
$$

Similarly, for any $E<5 / 2$, the limit of the other denominator is given by:

$$
\frac{1}{2 \beta}+\frac{1}{2 \pi^{3 / 2}} \int_{0}^{1} \frac{\xi^{1 / 2}\left[1-\xi^{-E}\left(e^{-x_{0}^{2} \frac{1-\xi}{1+\xi}}-e^{-x_{0}^{2} \frac{1+\xi}{1-\xi}}\right)\right]}{\left(1-\xi^{2}\right)^{3 / 2}} d \xi<\infty
$$

taking into account that, as pointed out in [11], we have:

$$
\xi^{-1}\left(e^{-x_{0}^{2} \frac{1-\xi}{1+\xi}}-e^{-x_{0}^{2} \frac{1+\xi}{1-\xi}}\right) \rightarrow 4 x_{0}^{2}
$$

as $\xi \rightarrow 0_{+}$, which implies that $\xi^{1 / 2-E}\left(e^{-x_{0}^{2} \frac{1-\xi}{1+\xi}}-e^{-x_{0}^{2} \frac{1+\xi}{1-\xi}}\right)$ has an integrable singularity at $0_{+}$ as long as $E<5 / 2$.

Hence, for any $x_{0}>0$ and any $E<3 / 2$ (resp. $E<5 / 2$ ), the difference of the two divergent series on the right hand side of (3.12) (resp. (3.12a)) yields a finite limit. Then, it is not difficult to prove that, as a consequence of the first resolvent identity, the same holds at any other point away from $\sigma\left(H_{0}\right)$. by:

Therefore, the norm resolvent limit of (3.7) after removing the ultraviolet cut-off is given

$$
\begin{aligned}
\left(H_{0}-E\right)^{-1}+\frac{\left|\left(H_{0}-E\right)_{s}^{-1}\left(\cdot, \vec{x}_{0}\right)\right\rangle\left\langle\left(H_{0}-E\right)_{s}^{-1}\left(\vec{x}_{0}, \cdot\right)\right|}{\frac{1}{2 \beta}+\lim _{\ell \rightarrow+\infty}\left[\frac{1}{2} \sum_{|\vec{n}|=0}^{\ell} \frac{\Psi_{2 \vec{n}}^{2}(0)}{|2 \vec{n}|+\frac{3}{2}}-\sum_{|\vec{n}|=0}^{\ell} \frac{\Psi_{2 \vec{n}}^{2}\left(\vec{x}_{0}\right)}{|2 \vec{n}|+\frac{3}{2}-E}\right]}+ \\
\frac{\left|\left(H_{0}-E\right)_{a s}^{-1}\left(\cdot, \vec{x}_{0}\right)\right\rangle\left\langle\left(H_{0}-E\right)_{a s}^{-1}\left(\vec{x}_{0}, \cdot\right)\right|}{\frac{1}{2 \beta}+\lim _{\ell \rightarrow+\infty}\left[\frac{1}{2} \sum_{|\vec{n}|=0}^{\ell} \frac{\Psi_{2 \vec{n}}^{2}(0)}{|2 \vec{n}|+\frac{3}{2}}-\sum_{|\vec{n}|=0}^{\ell} \frac{\psi_{2 n_{1}+1}^{2}\left(x_{0}\right) \psi_{2 n_{2}}^{2}(0) \psi_{2 n_{3}}^{2}(0)}{\left.2 n_{1}+2 n_{2}+2 n_{3}+\frac{5}{2}-E\right]}\right.} .
\end{aligned}
$$


In particular, for any $E<3 / 2$, we have the following integral representation:

$$
\begin{aligned}
& \left(H_{0}-E\right)^{-1}+\frac{\left|\left(H_{0}-E\right)_{s}^{-1}\left(\cdot, \vec{x}_{0}\right)\right\rangle\left\langle\left(H_{0}-E\right)_{s}^{-1}\left(\vec{x}_{0}, \cdot\right)\right|}{\frac{1}{2 \beta}+\frac{1}{2 \pi^{3 / 2}} \int_{0}^{1} \frac{\xi^{1 / 2}\left[1-\xi^{-E}\left(e^{-x_{0}^{2} \frac{1-\xi}{1+\xi}}+e^{-x_{0}^{2} \frac{1+\xi}{1-\xi}}\right)\right]}{\left(1-\xi^{2}\right)^{3 / 2}} d \xi}+ \\
& \frac{\left|\left(H_{0}-E\right)_{a s}^{-1}\left(\cdot, \vec{x}_{0}\right)\right\rangle\left\langle\left(H_{0}-E\right)_{a s}^{-1}\left(\vec{x}_{0}, \cdot\right)\right|}{\frac{1}{2 \beta}+\frac{1}{2 \pi^{3 / 2}} \int_{0}^{1} \frac{\xi^{1 / 2}\left[1-\xi^{-E}\left(e^{-x_{0}^{2} \frac{1-\xi}{1+\xi}}-e^{-x_{0}^{2} \frac{1+\xi}{1-\xi}}\right)\right]}{\left(1-\xi^{2}\right)^{3 / 2}} d \xi} .
\end{aligned}
$$

By essentially mimicking the proofs used in some of the above-mentioned papers $[2,9$, 17], it can be rigorously shown that the latter operator is indeed the resolvent of a semibounded self-adjoint operator for any $E$ below $E_{0}(\beta)$, the lowest zero of the denominator in the second term in (3.14) and (3.14a). Furthermore, the explicit form of the resolvent clearly implies its analyticity in norm in close proximity to 0 (since we have an analytic family in the sense of Kato, see $[14,21])$ as a function of $\beta$.

The results obtained so far can thus be summarized in the following theorem.

Theorem 3.1. The rigorous Hamiltonian of the three-dimensional isotropic oscillator, perturbed by two identical attractive point interactions situated symmetrically with respect to the origin at the points $\pm \vec{x}_{0}=\left( \pm x_{0}, 0,0\right), x_{0}>0$, making sense of the merely formal expression (3.1), is the self-adjoint operator $H_{\left\{\beta, \vec{x}_{0}\right\}}$ whose resolvent is given by the bounded operator (3.14). The latter is the limit of the resolvents (3.7) (of the Hamiltonians with the energy cut-off defined by (3.2)) in the norm topology of bounded operators on $L_{2}\left(\mathbb{R}^{3}\right)$ once the energy cut-off is removed. Furthermore, $H_{\left\{\beta, \vec{x}_{0}\right\}}$, regarded as a function of $\beta$, is an analytic family in the sense of Kato.

Of course, unlike the Hamiltonian analyzed in Section 2, we can no longer hope to have the bound state equation expressed in terms of a ratio of Gamma functions.

Nevertheless, since the limit of the difference of the divergent series appearing in the first (resp. second) denominator of (3.14) gives rise to a smooth multibranch function of the energy parameter, with its vertical asymptotes given by the symmetric (resp. antisymmetric) eigenvalues of the unperturbed Hamiltonian, we can rely on the findings of [8-11] to have it expressed in terms of an integral that will have to be suitably modified for each single level in order to avoid the unpleasant appearance of divergences.

Remark. In perfect accordance with what was observed in [10] for the analogous one-dimensional model with a single point impurity centered away from the origin, and in [11] for the model having a pair of twin impurities symmetrically situated with respect to the origin, it is worth pointing out that whenever the location of the impurities coincides with a node of an eigenfunction for the one-dimensional harmonic oscillator (here we are obviously excluding the trivial case $x_{0}=0$ ), then the corresponding eigenenvalue will not be affected at all by the singular perturbation. In other words, if

$$
\left.\psi_{2 m_{1}}\left(x_{0}\right)=0, \quad x_{0}>0 \quad \text { (respectively } \psi_{2 m_{1}+1}\left(x_{0}\right)=0\right)
$$

then

$$
\Psi_{2 \vec{m}}(\vec{x}), \quad \vec{m}=\left(m_{1}, m_{2}, m_{3}\right) \quad\left(\text { resp. } \psi_{2 m_{1}+1}(x) \psi_{2 m_{2}}(y) \psi_{2 m_{3}}(z) t\right)
$$


Spectral properties of a symmetric three-dimensional quantum dot ...

belongs to the kernel of the first (resp. second) rank-one operator in (3.14) and the corresponding eigenvalue

$$
|2 \vec{m}|+\frac{3}{2} \quad\left(\text { resp. } 2 m_{1}+2 m_{2}+2 m_{3}+\frac{5}{2}\right)
$$

will stay in the spectrum with its original multiplicity.

Hence, for any $E<3 / 2$, the equation determining the ground state energy is given by:

$$
\frac{1}{\beta}=\frac{1}{\pi^{3 / 2}} \int_{0}^{1} \frac{\xi^{1 / 2}\left[\xi^{-E}\left(e^{-x_{0}^{2} \frac{1-\xi}{1+\xi}}+e^{-x_{0}^{2} \frac{1+\xi}{1-\xi}}\right)-1\right]}{\left(1-\xi^{2}\right)^{3 / 2}} d \xi .
$$

By setting $\alpha=1 / \beta$ and

$$
\alpha_{0}^{+}\left(x_{0}\right)=\frac{1}{\pi^{3 / 2}} \int_{0}^{1} \frac{\left(e^{-x_{0}^{2} \frac{1-\xi}{1+\xi}}+e^{-x_{0}^{2} \frac{1+\xi}{1-\xi}}\right)-\xi^{1 / 2}}{\left(1-\xi^{2}\right)^{3 / 2}} d \xi<\infty,
$$

the equation can be exactly recast in a manner that is completely analogous to (2.5d), that is to say:

$$
\alpha-\alpha_{0}^{+}\left(x_{0}\right)=\frac{1}{\pi^{3 / 2}} \int_{0}^{1} \frac{\left(\xi^{1 / 2-E}-1\right)\left(e^{-x_{0}^{2} \frac{1-\xi}{1+\xi}}+e^{-x_{0}^{2} \frac{1+\xi}{1-\xi}}\right)}{\left(1-\xi^{2}\right)^{3 / 2}} d \xi .
$$

The graph for the solution of the above equation, i.e. the ground state energy as a function of the extension parameter, is shown below in Fig. 3 for $x_{0}=0.2$.

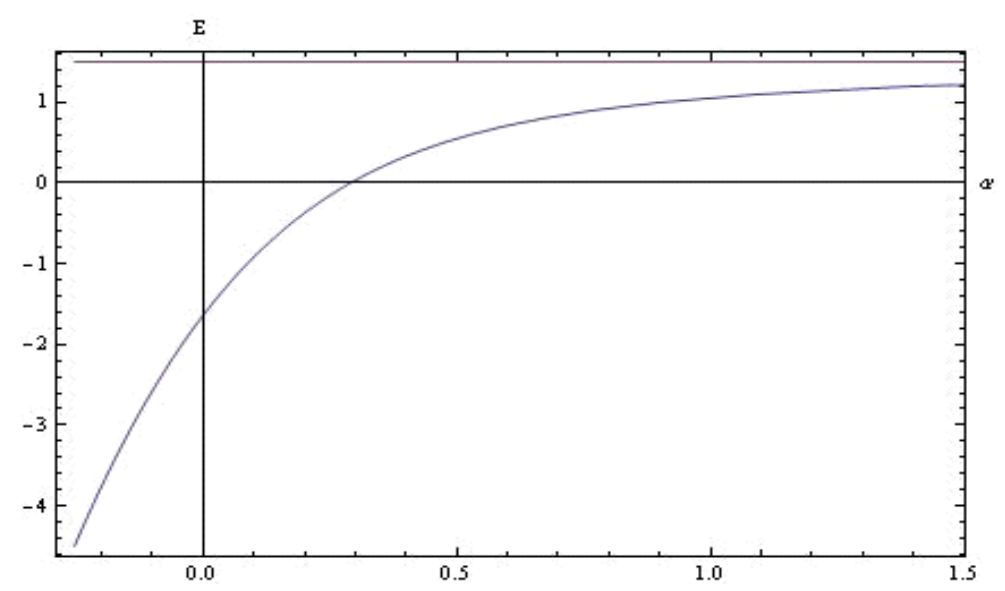

FIG. 3. The ground state energy for the 3D-isotropic harmonic oscillator perturbed by a pair of identical attractive point interactions symmetrically situated at a distance $x_{0}=0.2$ from the origin as a function of the extension parameter $\alpha=1 / \beta$

Similarly, the equation determining the energy of the lowest antisymmetric bound state for any $E<5 / 2$, namely:

$$
\frac{1}{\beta}=\frac{1}{\pi^{3 / 2}} \int_{0}^{1} \frac{\xi^{1 / 2}\left[\xi^{-E}\left(e^{-x_{0}^{2} \frac{1-\xi}{1+\xi}}-e^{-x_{0}^{2} \frac{1+\xi}{1-\xi}}\right)-1\right]}{\left(1-\xi^{2}\right)^{3 / 2}} d \xi
$$


after setting

$$
\alpha_{0}^{-}\left(x_{0}\right)=\frac{1}{\pi^{3 / 2}} \int_{0}^{1} \frac{\left(e^{-x_{0}^{2} \frac{1-\xi}{1+\xi}}-e^{-x_{0}^{2} \frac{1+\xi}{1-\xi}}\right)-\xi^{1 / 2}}{\left(1-\xi^{2}\right)^{3 / 2}} d \xi<\infty,
$$

can be recast as:

$$
\alpha-\alpha_{0}^{-}\left(x_{0}\right)=\frac{1}{\pi^{3 / 2}} \int_{0}^{1} \frac{\left(\xi^{1 / 2-E}-1\right)\left(e^{-x_{0}^{2} \frac{1-\xi}{1+\xi}}-e^{-x_{0}^{2} \frac{1+\xi}{1-\xi}}\right)}{\left(1-\xi^{2}\right)^{3 / 2}} d \xi .
$$

The graph for the solution of the above equation, representing the energy of the lowest antisymmetric bound state as a function of the extension parameter, is shown below in Fig. 4 for $x_{0}=0.2$.

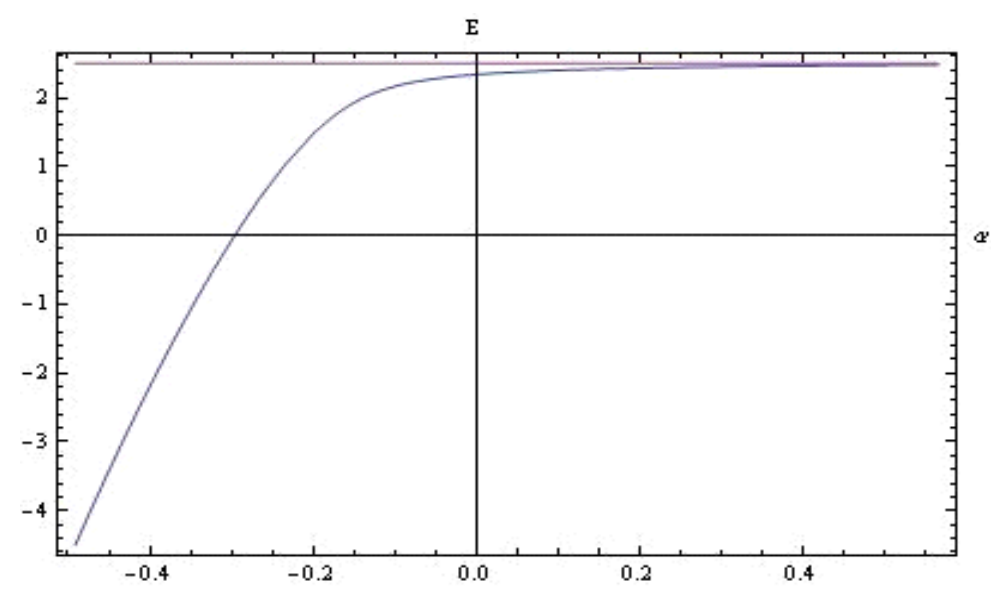

FIG. 4. The energy for the lowest antisymmetric bound state of the 3D-isotropic harmonic oscillator perturbed by a pair of identical attractive point interactions symmetrically situated at a distance $x_{0}=0.2$ from the origin as a function of the extension parameter $\alpha=1 / \beta$

By further exploiting the technique developed in the aforementioned papers [8-11] to obtain cancellation of the divergences as we move to the right of $E=3 / 2$, we can also study the equation determining the second symmetric bound state energy.

Given that:

$$
\begin{aligned}
\lim _{\ell \rightarrow+\infty}\left[\frac{1}{2} \sum_{|\vec{n}|=0}^{\ell} \frac{\Psi_{2 \vec{n}}^{2}(0)}{|2 \vec{n}|+\frac{3}{2}}-\sum_{|\vec{n}|=0}^{\ell} \frac{\Psi_{2 \vec{n}}^{2}\left(\vec{x}_{0}\right)}{|2 \vec{n}|+\frac{3}{2}-E}\right]= \\
\\
\quad \frac{1}{2} \frac{\Psi_{0}^{2}(0)}{3 / 2}-\frac{\Psi_{0}^{2}\left(\vec{x}_{0}\right)}{3 / 2-E}+\lim _{\ell \rightarrow+\infty}\left[\frac{1}{2} \sum_{|\vec{n}|=1}^{\ell} \frac{\Psi_{2 \vec{n}}^{2}(0)}{|2 \vec{n}|+\frac{3}{2}}-\sum_{|\vec{n}|=1}^{\ell} \frac{\Psi_{2 \vec{n}}^{2}\left(\vec{x}_{0}\right)}{\left.|2 \vec{n}|+\frac{3}{2}-E\right]}\right.
\end{aligned}
$$

and

$$
\frac{\Psi_{0}^{2}(0)}{3 / 2}=\frac{1}{\pi^{3 / 2}} \int_{0}^{1} \xi^{1 / 2} d \xi, \frac{\Psi_{0}^{2}\left(x_{0}\right)}{3 / 2-E}=\frac{e^{-x_{0}^{2}}}{\pi^{3 / 2}} \int_{0}^{1} \xi^{1 / 2-E} d \xi
$$


the equation determining the second symmetric bound state energy is given by:

$$
\begin{aligned}
\frac{1}{\beta}=\frac{2 e^{-x_{0}^{2}}}{(3 / 2-E) \pi^{3 / 2}}-\frac{2}{3 \pi^{3 / 2}}+\frac{1}{\pi^{3 / 2}} \int_{0}^{1} \frac{\xi^{1 / 2}\left[\left(1-\xi^{2}\right)^{3 / 2}-1\right]}{\left(1-\xi^{2}\right)^{3 / 2}} d \xi+ \\
\frac{1}{\pi^{3 / 2}} \int_{0}^{1} \frac{\xi^{1 / 2-E}\left[e^{-x_{0}^{2}(1-\xi) /(1+\xi)}+e^{-x_{0}^{2}(1+\xi) /(1-\xi)}-2 e^{-x_{0}^{2}}\left(1-\xi^{2}\right)^{3 / 2}\right]}{\left(1-\xi^{2}\right)^{3 / 2}} d \xi .
\end{aligned}
$$

Since the quantity

$$
e^{-x_{0}^{2}(1-\xi) /(1+\xi)}+e^{-x_{0}^{2}(1+\xi) /(1-\xi)}-2 e^{-x_{0}^{2}}\left(1-\xi^{2}\right)^{3 / 2}
$$

behaves like $c \xi^{2}+O\left(\xi^{4}\right)$ in the region to the right of the origin, it is quite evident that the numerator inside the second integral on the right hand side of (3.18) has an integrable singularity at the lower limit of integration for any $E<7 / 2$, that is to say the third eigenvalue of the unperturbed 3D-isotropic harmonic oscillator. One can immediately notice that both integrals on the right hand side of (3.18) diverge as $\xi \rightarrow 1_{-}$. However, by rewriting their sum as:

$$
\frac{1}{\pi^{3 / 2}} \int_{0}^{1} \frac{\xi^{1 / 2}\left\{\left(1-\xi^{2}\right)^{3 / 2}-1-\xi^{-E}\left[2 e^{-x_{0}^{2}}\left(1-\xi^{2}\right)^{3 / 2}-e^{-x_{0}^{2}(1-\xi) /(1+\xi)}-e^{-x_{0}^{2}(1+\xi) /(1-\xi)}\right]\right\}}{\left(1-\xi^{2}\right)^{3 / 2}} d \xi
$$

it is a bit tedious but straightforward to check that:

$$
\frac{\xi^{1 / 2}\left\{\left(1-\xi^{2}\right)^{3 / 2}-1-\xi^{-E}\left[2 e^{-x_{0}^{2}}\left(1-\xi^{2}\right)^{3 / 2}-e^{-x_{0}^{2}(1-\xi) /(1+\xi)}-e^{-x_{0}^{2}(1+\xi) /(1-\xi)}\right]\right\}}{1-\xi^{2}} \rightarrow L_{E}<\infty
$$

as $\xi \rightarrow 1_{-}$, which implies the integrable nature of the singularity at the upper limit of integration and, therefore, the cancellation of the two divergent quantities on the right hand side of (3.18). Hence, for any $3 / 2<E<7 / 2$, the equation determining the second symmetric bound state energy can be recast as:

$$
\begin{aligned}
& \frac{1}{\beta}=\frac{2 e^{-x_{0}^{2}}}{(3 / 2-E) \pi^{3 / 2}}-\frac{2}{3 \pi^{3 / 2}}+\frac{1}{\pi^{3 / 2}} \times \\
& \int_{0}^{1} \frac{\xi^{1 / 2}\left\{\left(1-\xi^{2}\right)^{3 / 2}-\xi^{-E}\left[2 e^{-x_{0}^{2}}\left(1-\xi^{2}\right)^{3 / 2}-e^{-x_{0}^{2}(1-\xi) /(1+\xi)}-e^{-x_{0}^{2}(1+\xi) /(1-\xi)}\right]\right\}}{\left(1-\xi^{2}\right)^{3 / 2}} d \xi .
\end{aligned}
$$

The latter equation can be further transformed into:

$$
\begin{aligned}
& \alpha-\alpha_{0}^{+}\left(x_{0}\right)=\frac{2 e^{-x_{0}^{2}}}{(3 / 2-E) \pi^{3 / 2}}-\frac{2}{3 \pi^{3 / 2}}+\frac{1}{\pi^{3 / 2}} \times \\
& {\left[\int_{0}^{1} \frac{\xi^{1 / 2}\left\{\left(1-\xi^{2}\right)^{3 / 2}-\xi^{-E}\left[2 e^{-x_{0}^{2}}\left(1-\xi^{2}\right)^{3 / 2}-e^{-x_{0}^{2}(1-\xi) /(1+\xi)}-e^{-x_{0}^{2}(1+\xi) /(1-\xi)}\right]\right\}}{\left(1-\xi^{2}\right)^{3 / 2}} d \xi+\right.} \\
& \left.\int_{0}^{1} \frac{-e^{-x_{0}^{2}(1-\xi) /(1+\xi)}-e^{-x_{0}^{2}(1+\xi) /(1-\xi)}}{\left(1-\xi^{2}\right)^{3 / 2}} d \xi\right] .
\end{aligned}
$$

Figure 5 shows the plot for the counterpart of Fig. 2 for $x_{0}=0.2$ with the five lowest eigenvalues, namely $E_{0}(\alpha), E_{1}(\alpha), E_{2}(\alpha)$ (clearly omitting their dependence on $x_{0}$ to make the 
notation less cumbersome), the new eigenenergies created by the pair of twin point interactions, in addition to $5 / 2$ and $7 / 2$, whose degeneracy has now been lowered to two and five respectively.

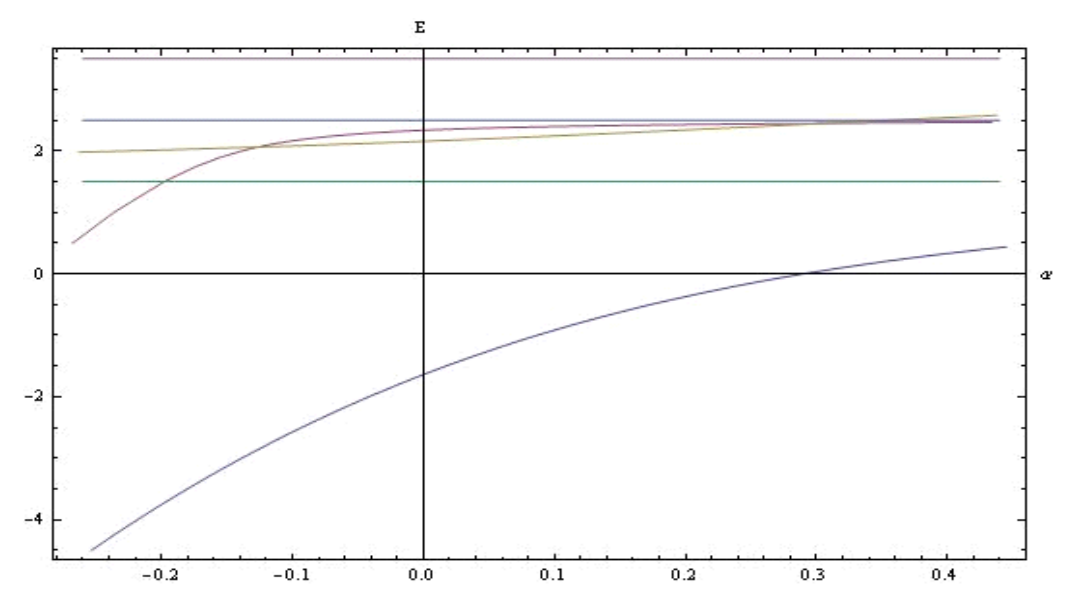

FIG. 5. The ground state energy and the next two eigenenergies created by two identical attractive $\delta$-perturbations situated symmetrically at the same distance from the origin, $x_{0}=0.2$, as functions of the extension parameter $\alpha=1 / \beta$

It is worth stressing that, in addition to level crossings of the same type as those encountered in the aforementioned articles [1,9], which occur in the case of a single $\delta$-impurity, involving the new eigenvalues created by the point perturbations and the eigenvalues of the unperturbed harmonic oscillator preserved under the perturbation due to their degeneracy, two further level crossings of a new type involving $E_{1}(\alpha)$ and $E_{2}(\alpha)$ (the two new energy levels created by the singular double-well perturbation above the ground state one), can be observed at the values $\alpha_{2}$ (approximately equal to -0.126478) and $\alpha_{3}$ (approximately equal to 0.309201). It seems rather remarkable to us that, for such a small value of the distance $x_{0}$, there is a range of values for the extension parameter $\alpha$, namely the interval $\left(\alpha_{2}, \alpha_{3}\right)$, inside which $E_{2}(\alpha)<E_{1}(\alpha)$. In other words, when the coupling is sufficiently strong (taking account of the fact that $\alpha=1 / \beta$ ) and the distance between the two centers is sufficiently small (this point will be made more precise shortly), the second symmetric bound state created by the singular perturbation can become more tightly bound than the lowest antisymmetric bound state. Furthermore, the energy level created by the perturbation at $\alpha_{2}$ (resp. $\left.\alpha_{3}\right)$ is doubly degenerate, since $E_{1}\left(\alpha_{2}\right)=E_{2}\left(\alpha_{2}\right)$ (resp. $E_{1}\left(\alpha_{3}\right)=E_{2}\left(\alpha_{3}\right)$ ).

In addition to the value $\alpha_{4}$ (approximately equal to 0.356236 ), where $E_{2}(\alpha)=5 / 2$, it is also worth including the value $\alpha_{1}$ (approximately equal to -0.198151 ) where $E_{1}(\alpha)=3 / 2$, even though $E=3 / 2$ is no longer an eigenvalue. Then, the spectral features displayed in Fig. 5 can be summarized in Table 1 .

Next, we will show how an increase in $x_{0}$ affects the energy levels considered before. As clearly shown by the comparison between the graph of the ground state energy as a function of $\alpha$ in Fig. 6 for $x_{0}=0.4$ and its analogue in Fig. 3 for $x_{0}=0.2$, we have $E_{0}(\alpha ; 0.2)<E_{0}(\alpha ; 0.4)$ for any $\alpha$.

Although we cannot strictly use the definition of "positional disorder" given in [1] for the 3D-isotropic quantum dot with a single impurity because of the presence of the vector $-\vec{x}_{0}$ in our model, we, nevertheless, believe that the concept is still valid in the sense that the greater the distance between the two impurities is, the less localized the ground state will be. It might be worth noting that this is in full agreement with our findings in some of the aforementioned papers [7, 11,17] on one-dimensional singular double wells (see also [10]). 
TABLE 1. The ordering of the five lowest eigenvalues over the corresponding five intervals of the extension parameter for $x_{0}=0.2$

\begin{tabular}{|c|c|}
\hline$\alpha<\alpha_{1}$ & $E_{0}(\alpha)<E_{1}(\alpha)<3 / 2<E_{2}(\alpha)<5 / 2$ \\
\hline$\alpha_{1} \leq \alpha<\alpha_{2}$ & $E_{0}(\alpha)<3 / 2 \leq E_{1}(\alpha)<E_{2}(\alpha)<5 / 2$ \\
\hline$\alpha_{2} \leq \alpha<\alpha_{3}$ & $E_{0}(\alpha)<3 / 2<E_{2}(\alpha) \leq E_{1}(\alpha)<5 / 2$ \\
\hline$\alpha_{3} \leq \alpha<\alpha_{4}$ & $E_{0}(\alpha)<3 / 2<E_{1}(\alpha) \leq E_{2}(\alpha)<5 / 2$ \\
\hline$\alpha_{4} \leq \alpha$ & $E_{0}(\alpha)<3 / 2<E_{1}(\alpha)<5 / 2 \leq E_{2}(\alpha)<7 / 2$ \\
\hline
\end{tabular}

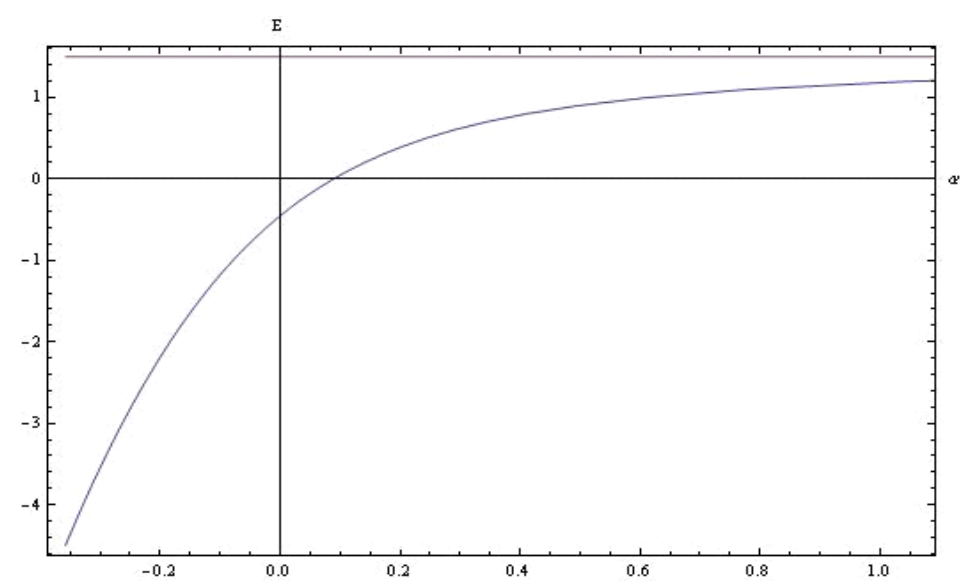

FIG. 6. The ground state energy of the 3D-isotropic harmonic oscillator perturbed by a pair of identical attractive point interactions symmetrically situated at a distance $x_{0}=0.4$ from the origin as a function of the extension parameter $\alpha=1 / \beta$

The graph of the lowest antisymmetric eigenvalue created by the singular double well when the separation distance becomes $x_{0}=0.4$ is also provided below (Fig.7).

We now wish to provide the analog of Fig. 5 for $x_{0}=0.3$ (Fig. 8).

As can be clearly seen in the plot, a remarkable change in the energy spectrum has now occurred: for any value of the extension parameter we have $E_{1}(\alpha)<E_{2}(\alpha)$, that is to say, the new energy levels created by the double well perturbation completely avoid each other, borrowing the terminology used in articles such as [22] on the spectral phenomenon called "level repulsion" or "avoided level crossing". Of course, we still have the level crossing between $E_{2}(\alpha)$ and $5 / 2$, the antisymmetric eigenvalue of the 3D-isotropic harmonic oscillator whose degeneracy has now been lowered to two by the perturbation.

The threshold value of $x_{0}$, denoted by $X_{t}$ in the following, can be determined by observing that for that particular value, the two spectral curves $E_{1}\left(\alpha, X_{t}\right)$ and $E_{2}\left(\alpha, X_{t}\right)$ must have the same value as well as the same partial derivative with respect to the extension parameter $\alpha$. Hence, $X_{t}$ is the second coordinate of the point representing the solution of the following 


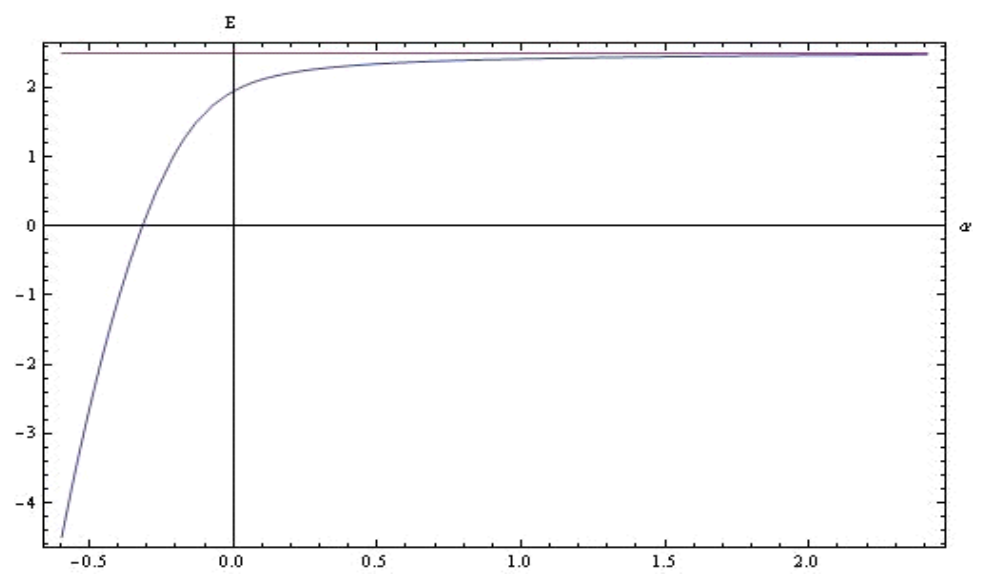

FIG. 7. The energy for the lowest antisymmetric bound state of the 3D-isotropic harmonic oscillator perturbed by a pair of identical attractive point interactions symmetrically situated at a distance $x_{0}=0.4$ from the origin as a function of the extension parameter $\alpha=1 / \beta$

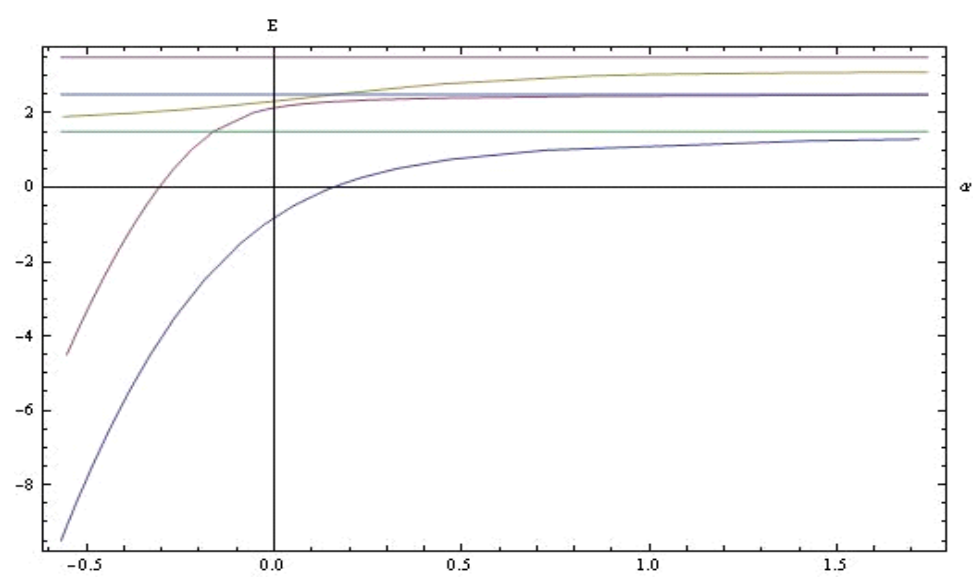

FIG. 8. The ground state energy and the next two eigenenergies created by two attractive $\delta$-perturbations situated symmetrically at the same distance from the origin, $x_{0}=0.3$, as functions of the extension parameter $\alpha=1 / \beta$

system:

$$
\left\{\begin{array}{c}
E_{1}\left(\alpha, x_{0}\right)=E_{2}\left(\alpha, x_{0}\right) ; \\
\frac{\partial}{\partial \alpha} E_{1}\left(\alpha, x_{0}\right)=\frac{\partial}{\partial \alpha} E_{2}\left(\alpha, x_{0}\right) .
\end{array}\right.
$$

For any fixed value of the distance $x_{0}$, the function $E_{1}\left(\alpha, x_{0}\right)$ with domain given by the entire real axis and range $(-\infty, 5 / 2)$ has its inverse given by $\alpha_{1}\left(E, x_{0}\right)$ with domain $(-\infty, 5 / 2)$ and range equal to the entire real axis.

Similarly, if we restrict $E_{2}\left(\alpha, x_{0}\right)$ to the domain $\left(-\infty, \alpha^{*}\right]$ and range $(-\infty, 5 / 2]$, with $E_{2}\left(\alpha^{*}, x_{0}\right)=5 / 2$, its inverse function is $\alpha_{2}\left(E, x_{0}\right)$ with domain $(-\infty, 5 / 2]$ and range $\left(-\infty, \alpha^{*}\right]$. Both functions can actually be written in terms of explicit integrals and functions:

$$
\alpha_{1}\left(E, x_{0}\right)=\frac{1}{\pi^{3 / 2}} \int_{0}^{1} \frac{\xi^{1 / 2}\left[\xi^{-E}\left(e^{-x_{0}^{2} \frac{1-\xi}{1+\xi}}-e^{-x_{0}^{2} \frac{1+\xi}{1-\xi}}\right)-1\right]}{\left(1-\xi^{2}\right)^{3 / 2}} d \xi
$$




$$
\begin{aligned}
& \alpha_{2}\left(E, x_{0}\right)=\frac{2 e^{-x_{0}^{2}}}{(3 / 2-E) \pi^{3 / 2}}-\frac{2}{3 \pi^{3 / 2}}+\frac{1}{\pi^{3 / 2}} \times \\
& \quad \int_{0}^{1} \frac{\xi^{1 / 2}\left\{\left(1-\xi^{2}\right)^{3 / 2}-1-\xi^{-E}\left[2 e^{-x_{0}^{2}}\left(1-\xi^{2}\right)^{3 / 2}-e^{-x_{0}^{2}(1-\xi) /(1+\xi)}-e^{-x_{0}^{2}(1+\xi) /(1-\xi)}\right]\right\}}{\left(1-\xi^{2}\right)^{3 / 2}} d \xi
\end{aligned}
$$

(of course, we could alternatively use (3.16b) and (3.18b)).

Consequently, also taking into account the well-known theorem on the derivative of the inverse function, instead of obtaining $X_{t}$ by solving the system (3.19), it is easier from a computational point of view to derive its value by solving the system:

$$
\left\{\begin{array}{c}
\alpha_{1}\left(E, x_{0}\right)=\alpha_{2}\left(E, x_{0}\right) \\
\frac{\partial}{\partial E} \alpha_{1}\left(E, x_{0}\right)=\frac{\partial}{\partial E} \alpha_{2}\left(E, x_{0}\right) .
\end{array}\right.
$$

The numerical solution of (3.19a) is the point with coordinates $E=E_{t}$ (approximately equal to 2.29949), $x_{0}=X_{t}$ (approximately equal to 0.2339644 ), where both functions $\alpha_{1}$ and $\alpha_{2}$ attain the value $\alpha_{t}$ (approximately equal to 0.0829). The plot of the tangential contact between the two spectral curves for $x_{0}=X_{t}$ is provided below in Fig. 9.

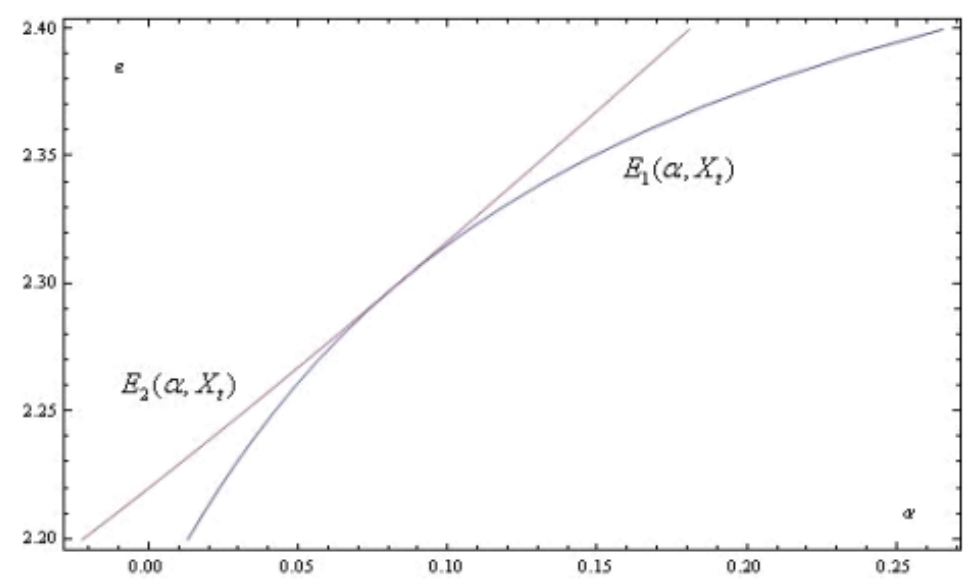

FIG. 9. The curves of the two eigenenergies $E_{1}\left(\alpha, X_{t}\right)$ and $E_{2}\left(\alpha, X_{t}\right)\left(X_{t}\right.$ being approximately equal to 0.2339644 ) intersecting each other tangentially at $\alpha=\alpha_{t}$ (approximately equal to 0.0829 )

Hence, what has been seen regarding the three different spectral configurations for the two energy levels $E_{1}\left(\alpha, x_{0}\right)$ and $E_{2}\left(\alpha, x_{0}\right)$ can be summarized by means of the following Table 2, which clearly shows the role played by the threshold value $x_{0}=X_{t}$.

The table thus makes our earlier statement written below Fig. 5 more precise: for any $x_{0}<X_{t}$, there exists an interval $\left(\alpha_{2}\left(x_{0}\right), \alpha_{3}\left(x_{0}\right)\right)$ (using the same indices used above) of values for the extension parameter $\alpha$ (or equivalently of its reciprocal $\beta$ ) for which $E_{2}\left(\alpha, x_{0}\right)<E_{1}\left(\alpha, x_{0}\right)$. In other words, as the values of $|\alpha|$ in that range are rather small $\left(\alpha_{2}(0.2)\right.$ approximately equal to -0.126478 and $\alpha_{3}(0.2)$ approximately equal to 0.309201 in our previous example) and the corresponding values of $|\beta|$ are large, such a singular configuration of those two eigenvalues (energy levels), characterized by having the second excited symmetric 
TABLE 2. The three possible configurations involving the two energy levels $E_{1}\left(\alpha, x_{0}\right)$ and $E_{2}\left(\alpha, x_{0}\right)$ as the distance parameter $x_{0}$ varies

\begin{tabular}{|c|c|}
\hline$x_{0}<X_{t} \cong 0.2339644$ & two distinct intersections between \\
& $E_{1}\left(\alpha, x_{0}\right)$ and $E_{2}\left(\alpha, x_{0}\right)$ \\
\hline$x_{0}=X_{t} \cong 0.2339644$, & one tangential intersection between \\
$\alpha_{t} \cong 0.0829$ & $E_{1}\left(\alpha, x_{0}\right)$ and $E_{2}\left(\alpha, x_{0}\right)$ \\
\hline$x_{0}>X_{t} \cong 0.2339644$ & no intersection since \\
& $E_{1}\left(\alpha, x_{0}\right)<E_{2}\left(\alpha, x_{0}\right)$ \\
\hline
\end{tabular}

bound state more tightly bound than the lowest antisymmetric one, can only occur by shrinking the distance between the two impurities below the threshold and raising their strength. Moreover, it can be seen that the interval expands as $x_{0}$ shrinks to zero.

Therefore, it might be fair to say that a wider separation of the two zero-range impurities makes the structure of the spectrum more similar to that of the unperturbed isotropic harmonic oscillator. As a matter of fact, it is not difficult to convince oneself that, for any fixed value of the coupling $\beta$ (or, equivalently, of the extension parameter $\alpha$ ), the denominators of both rank-one operators in (3.14) or (3.14a) are bound to diverge as $x_{0} \rightarrow+\infty$. Hence, the resolvent of our Hamiltonian will approach that of the 3D-isotropic harmonic oscillator in the limit $x_{0} \rightarrow+\infty$. As a result, that is in perfect agreement with its one-dimensional counterpart established in Theorem 2.2(a) in [11].

However, a sharp contrast with the one-dimensional model occurs for the opposite limit analyzed in Theorem 2.2(b) in [11]. In fact, by noting that the right hand sides of both (3.15) and (3.16) become divergent as $x_{0} \rightarrow 0_{+}$, it is clear that the lowest eigenvalue of our Hamiltonian will decrease without any lower bound as the distance shrinks to zero. Therefore, in sharp contrast with the one-dimensional case, we cannot expect in the limit $x_{0} \rightarrow 0_{+}$to obtain the operator studied in Section 2 with the strength of the point interaction doubled. It might also be worth reminding the reader that, as is well known to Quantum Chemistry students, three-dimensional interactions with a nonzero range do not manifest this singular behavior as the distance between the two centers shrinks to zero, as the classical textbook example of $\mathrm{H}_{2}^{+}$ smoothly approaching $\mathrm{He}^{+}$in the limit $R \rightarrow 0_{+}$clearly shows (see [23-25]).

The same singular spectral phenomenon has been recently observed in [17] where another model requiring a renormalization procedure, that is to say the one-dimensional Salpeter Hamiltonian with a double well of identical attractive point interactions, was investigated. Our spectral finding in this work confirms the general conjecture formulated in the above-mentioned paper that, when we deal with point interactions requiring the renormalization of the coupling constant (either because of the higher dimension or because of the different kinetic term), we must inevitably give up "the intuitive idea that in the limit $\left(x_{0} \rightarrow 0_{+}\right)$the eigenvalue should approach the ground state energy pertaining to a single delta with double strength".

In analogy with what was proved in [17], it will be shown in a forthcoming paper that the only possible way of obtaining the expected behavior in the limit also in the case of the operator investigated in this work is to make the coupling constant $\mu(\ell, \beta)$ used in the renormalization procedure suitably dependent on the separation distance $x_{0}$. 


\section{Conclusions and perspectives}

After reviewing the spectral features of a three-dimensional isotropic quantum dot with an attractive point impurity situated at the origin (the bottom of the confining harmonic potential), a model investigated in papers such as $[1,9,26]$, the model in which a pair of twin attractive point impurities gets inserted into the 3D-isotropic quantum dot has been studied by first defining in a rigorous manner the self-adjoint Hamiltonian representing the energy operator for such a model. Since the energy eigenvalues are given by the poles of the resolvent, its explicit expression has enabled us to start the spectral analysis of the model. Although the bound state equation no longer has the simple expression involving a ratio of Gamma functions, as was the case in the presence of a single impurity centered at the origin, it is still possible to carry out, at least conceptually, the required spectral analysis.

Here, we have restricted our task to a rather detailed description of the behavior of the lowest lying spectrum eigenvalues (energy levels) with respect to variations of the two key parameters of the model, one labeling the self-adjoint extensions of the operator (or its reciprocal) and the other one given by the distance between either impurity and the origin.

The important spectral phenomenon called "positional disorder" singled out in [1] (see also the references cited therein as well as [10]) in the case of a single point impurity located away from the origin retains its validity in the sense that the more separated the twin impurities are the less localized the ground state is.

In addition to the level crossings involving the spectral curve of each new singular perturbation-created eigenenergy and the next lower eigenvalue of the unperturbed harmonic oscillator (still present in the spectrum because of its degeneracy), another seemingly more noteworthy spectral phenomenon occurs. There exists a threshold for the separation distance between the twin impurities, above which, the spectral curve $E_{2}\left(\alpha, x_{0}\right)$, representing the energy of the second symmetric bound state, lies entirely above $E_{1}\left(\alpha, x_{0}\right)$, the curve pertaining to the energy for the lowest antisymmetric bound state. Below that threshold, there exists a range of values of the extension parameter, which is physically characterized by being proportional to the inverse scattering length, over which $E_{2}\left(\alpha, x_{0}\right) \leq E_{1}\left(\alpha, x_{0}\right)$, implying the existence of two new types of level crossings. From the point of view of what could be called "spectral engineering", the latter implies that the second symmetric bound state can become more tightly bound than the lowest antisymmetric bound state provided the separation distance is below the threshold and the extension parameter is sufficiently small. At the threshold value we have shown the existence of a single tangential intersection between the two spectral curves. As a consequence, the narrower the separation between the impurities is, the wider the aforementioned range is.

The current analysis could be further extended to the next higher eigenvalues to explore the existence of other distance thresholds leading to level crossings of the new type.

It has also been pointed out that, in full accordance with the findings of [17] for another singular double well model requiring renormalization of the coupling constant, and in total contrast to those of [11] for the one-dimensional counterpart of the model studied in this note, not requiring the renormalization procedure, the limit of our Hamiltonian as the separation distance shrinks to zero is not given by the Hamiltonian of the 3D-isotropic quantum dot with a single point impurity centered at the origin having twice the strength. It is our intention to prove in a forthcoming paper that the expected behavior in the limit can be achieved by making the coupling constant used in the renormalization procedure also dependent on the separation distance.

Finally, we also intend to extend our investigation to the two-dimensional counterpart of the model given the growing relevance of that dimension in Nanophysics, as attested by some 
contributions to the relevant literature, such as [27-30]. Of course, as can be seen in the case of the 2D-isotropic quantum dot with a single impurity, fully studied in [31], some logarithmic divergences, which are typical of two-dimensional Quantum Mechanics, are inevitably bound to arise.

\section{Acknowledgments}

Silvestro Fassari and Fabio Rinaldi wish to express their heartfelt gratitude to Prof. I. Popov and Prof. I. Lobanov for their kind invitation to present the main contents of this note during one of the sessions of the conference "Mathematical Challenge of Quantum Transport in Nanosystems", held at ITMO University, St. Petersburg, Russian Federation (9 - 11 September 2015).

Sergio Albeverio is very grateful to Prof. Nicolas Monod, Director of "Centre Interfacultaire Bernoulli - EPFL", Lausanne, for the warm hospitality during his stay there as organiser jointly with Prof. A. B. Cruzeiro and Prof. D. Holm of the semester programme "Geometric Mechanics, Variational and Stochastic Methods" (January - June 2015).

S. Fassari and F. Rinaldi also wish to thank Prof. A. B. Cruzeiro and Prof. J. C. Zambrini for their warm hospitality on the occasion of their visit to CIB - EPFL.

\section{References}

[1] Brüning J., Geyler V., Lobanov I. Spectral properties of a short-range impurity in a quantum dot. Journal of Mathematical Physics, 2004, 45, P. 1267-1290.

[2] Albeverio S., Fassari S., Rinaldi F. A remarkable spectral feature of the Schrödinger Hamiltonian of the harmonic oscillator perturbed by an attractive $\delta$ '-interaction centred at the origin: double degeneracy and level crossing. Journal of Physics A: Mathematical and Theoretical, 2013, 46(38), 385305.

[3] Albeverio S., Fassari S., Rinaldi F. The Hamiltonian of the harmonic oscillator with an attractive $\delta$ '-interaction centred at the origin as approximated by the one with a triple of attractive $\delta$-interactions. Journal of Physics A: Mathematical and Theoretical, 2016, 49(2), 025302.

[4] Albeverio S., Gesztesy F., Høegh-Krohn R., Holden H. Solvable models in Quantum Mechanics. AMS (Chelsea Series) second edition (with an appendix by P. Exner), 2004.

[5] Albeverio S., Kurasov P. Singular Perturbations of Differential Operators: Solvable Type Operators. Cambridge University Press, 2000.

[6] Albeverio S., Dabrowski L., Kurasov P. Symmetries of Schrödinger operators with point interactions. Letters in Mathematical Physics, 1998, 45, P. 33-47.

[7] Fassari S., Rinaldi F. On the spectrum of the Schrödinger Hamiltonian with a particular configuration of three point interactions. Reports on Mathematical Physics, 2009, 64(3), P. 367-393.

[8] Fassari S., Inglese G. On the spectrum of the harmonic oscillator with a $\delta$-type perturbation. Helvetica Physica Acta,1994, 67, P. 650-659.

[9] Fassari S., Inglese G. Spectroscopy of a three-dimensional isotropic harmonic oscillator with a $\delta$-type perturbation. Helvetica Physica Acta, 1996, 69, P. 130-140.

[10] Fassari S., Inglese G. On the spectrum of the harmonic oscillator with a $\delta$-type perturbation II. Helvetica Physica Acta, 1997, 70, P. 858-865.

[11] Fassari S., Rinaldi F. On the spectrum of the Schrödinger Hamiltonian of the one-dimensional harmonic oscillator perturbed by two identical attractive point interactions. Reports on Mathematical Physics, 2012 , 69(3), P. 353-370.

[12] Plancherel M., Rotach W. Sur les valeurs asymptotiques des polynomes d'Hermite. Commentarii Mathematici Helvetici, 1929, 1(1), P. 227-254.

[13] Mityagin B., Siegl P. Root system of singular perturbations of the harmonic oscillator type operators. ArXiv: 1307.6245, 2013.

[14] Reed M., Simon B. Analysis of Operators - Methods of Modern Mathematical Physics IV, Academic Press NY, 1978.

[15] Avakian M.P., Pogosyan G.S., Sissakian A.N., Ter-Antonyan V.M. Spectroscopy of a singular linear oscillator. Physics Letters A, 1987, 124( 4-5), P. 233-236. 
[16] Reed M., Simon B. Fourier Analysis, Self-adjointness - Methods of Modern Mathematical Physics II, Academic Press NY, 1975.

[17] Albeverio S., Fassari S., Rinaldi F. The discrete spectrum of the spinless Salpeter Hamiltonian perturbed by $\delta$-interactions. Journal of Physics A: Mathematical and Theoretical, 2015, 48(18), 185301.

[18] Reed M., Simon B. Functional Analysis - Methods of Modern Mathematical Physics I, Academic Press NY, 1972.

[19] Klaus M. A remark about weakly coupled one-dimensional Schrödinger operators. Helvetica Physica Acta, 1979, 52, P. 223-229.

[20] Fassari S. An estimate regarding one-dimensional point interactions. Helvetica Physica Acta, 1995, 68, P. $121-125$.

[21] Kato T. Perturbation Theory for Linear Operators, Springer Verlag Heidelberg-NY, 1995.

[22] Demkov Yu.N., Kurasov P.B. Von Neumann-Wigner Theorem: level repulsion and degenerate eigenvalues. Theoretical and Mathematical Physics, 2007, 153(1), P. 1407-1422.

[23] Schmidtke H.-H. Quantenchemie Weinheim: VCH, 1987 (in German).

[24] Byers Brown W., Steiner E. On the electronic energy of a one-electron diatomic molecule near the united atom. Journal of Chemical Physics, 1966, 44, P. 3934.

[25] Klaus M. On $\mathrm{H}_{2}^{+}$for small internuclear separation. Journal of Physics A: Mathematical and General, 1983, 16, P. 2709-2720.

[26] Gonzalez-Santander C., Dominguez-Adame F. Non-local separable solutions of two interacting particles in a harmonic trap. Physics Letters A, 2011, 375, P. 314-317.

[27] Gonzalez-Santander C., Dominguez-Adame F. Exciton states and optical absorption in quantum wires under laser radiation. Physics Letters A, 2010, 374, P. 2259-2261.

[28] Gonzalez-Santander C., Dominguez-Adame F. Modelling of Coulomb interaction in parabolic quantum wires. Physica E, 2009, 41, P. 1645-1647.

[29] Lima R.P.A., Amado M., Dominguez-Adame F. A solvable model of hydrogenic impurities in quantum dots. Nanotechnology, 2008, 19, P. 135402-5406.

[30] Lopez S., Dominguez-Adame F. Non-local potential approach to the ground state of confined excitons in quantum dots. Semiconductor Science Technology, 2002, 17, P. 227-229.

[31] Tusek M. Analysis of Two-dimensional Quantum Models with Singular Potentials. Diploma Thesis Czech Technical University in Prague, Faculty of Nuclear Sciences and Physical Engineering, 2006. 